\title{
Development of the adjusting and inspecting equipment for EDM Haipeng Huang ${ }^{1, a}$, Maosheng $\mathrm{Li}^{2, \mathrm{~b}}$, Guanxin $\mathrm{Chi}^{2, \mathrm{c}}$, Zhenlong Wang ${ }^{2, \mathrm{~d}}$ \\ ${ }^{1}$ School of Mechanical and Automotive Engineering, Xiamen University of Technology, Xiamen, 361024, China \\ ${ }^{2}$ Department of Mechanical Engineering, Harbin Institute of Technology, Harbin, 150001, China \\ aemail: huanghaipeng@xmut.edu.cn, bemail: 27041109@qq.com, \\ cemail: 114616921@qq.com, demail: wangzl@hit.edu.cn
}

Keywords: Adjust; Inspect; EDM

Abstract. According to the problem that lots of machining time of Electronic Discharge Machining (short for EDM) is wasted in replacing and inspecting tool-electrode, adjusting and inspecting equipment for EDM has been developed. Through extracting the feature points and planning the inspecting path, necessary data can be gotten. With accuracy evaluation method, the accuracy and the eligibility of the tool-electrode can be determined. With the principle of adjusting function, the initial position of the tool-electrode can be adjusted. By proving experimentation, this equipment can satisfy the demand of adjusting and inspecting of the tool-electrode of EDM.

\section{Introduction}

EDM is a kind of non-traditional machining. Work piece and tool-electrode are installed in the two poles respectively. The discharge between the two poles eclipses the work piece to complete the machining [1]. The machining type of EDM is shape-copying. The tool-electrode is designed specially according to the surface form of the aiming products [2]. In order to guarantee the machining accuracy, the tool-electrode must have high shape accuracy, and its initial position must be accurate [3]. During the machining process, the wastage of the tool-electrode is very high, and the tool-electrode has to be replaced frequently [4]. The initial position of the tool-electrode has to be adjusted every time [5]. During the process of adjusting, the machining has to be stopped. This wastes lots of machining time. So it is necessary to develop a adjusting and inspecting equipment. With this equipment, at the same time of the machining, the adjusting and inspecting of the tool-electrode can be accomplished in sequence. When the damaged tool-electrode has to be replaced, the one that has adjusted completely can be installed on the machine directly.

\section{The components of the equipment}

The model of the equipment designed by Solidworks and the real product photo are shown in figure 1.

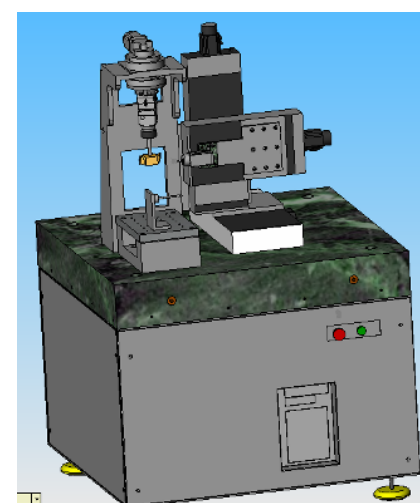

(a) Model of the equipment

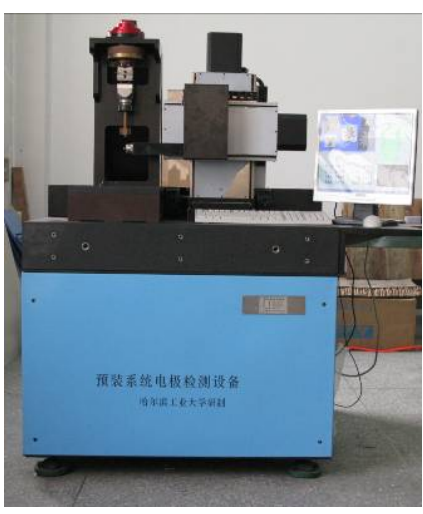

(b) Real product photo

Fig.1. The components of the equipment 
There are 4 linkage axes in this equipment. $\mathrm{X}$ axis, $\mathrm{Y}$ axis and $\mathrm{Z}$ axis are liner axes, and $\mathrm{C}$ axis is rotating axis. $\mathrm{X}$ axis, $\mathrm{Y}$ axis and $\mathrm{Z}$ axis are installed on the right side of the equipment and the probe is installed on $\mathrm{X}$ axis. $\mathrm{C}$ axis is installed on the left side of the equipment and a standard system 3R fixture is installed on it. The tool-electrode is installed on the fixture. During the working procedure, $\mathrm{C}$ axis adjusts the pose of the tool-electrode, other 3 axes drive probe to measure the coordinate information of the tool-electrode. According the coordinate information, the tool-electrode can be adjusted and inspected.

\section{The principle of adjusting function}

The adjusting procedure is shown in figure 2.

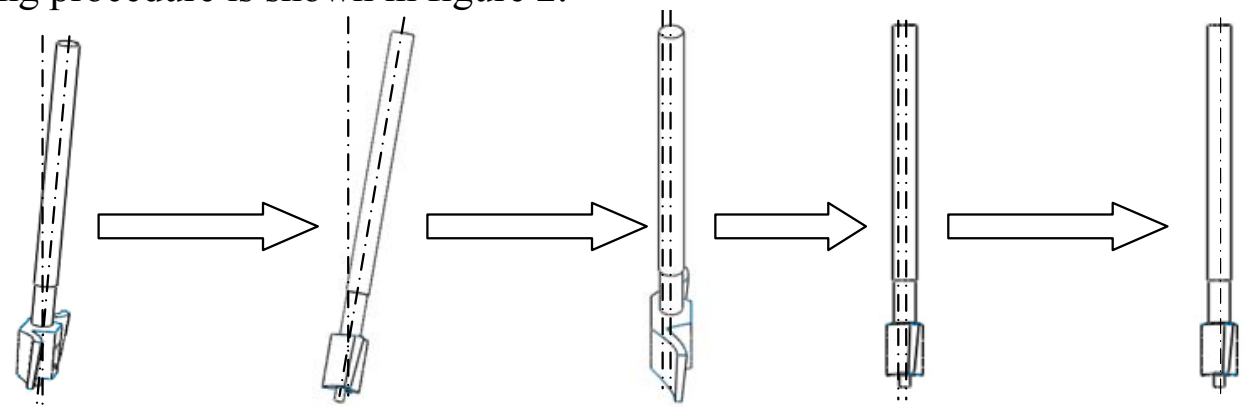

Fig.2. The adjusting procedure

The procedure can be divided into 4 steps:

(1) Adjusting the error rolling $\mathrm{Z}$ axis: measuring some points' coordinate information along the $\mathrm{Y}$ direction of the reference plane to determine the rotating degree.

(2) Adjusting the error rolling Y axis: measuring some points' coordinate information of 2 lines along the $\mathrm{Z}$ direction of the reference plane to determine the rotating degree.

(3) Adjusting the error rolling $X$ axis: measuring some points' coordinate information along the $Z$ direction of the reference plane to determine the rotating degree.

(4) Adjusting the coaxiality error between the axial line of the tool-electrode and $\mathrm{C}$ axis: measuring the section of the tool-electrode's installing pole to determine the displacement.

Through repeating above steps, the initial position of the tool-electrode can be adjusted into the margin of error. When the damaged tool-electrode has to be replaced, one that has been adjusted can be installed on the machine with standard system 3R fixture. Then the machining can continue immediately. With this function, the adjusting work is changed into off-line form from on-line form; the adjusting and machining are changed into parallel relationship form serial relationship.

\section{Extracting the feature points of the tool-electrode}

Because the data information of the tool-electrode's surfaces is very large, extracting the feature points of the tool-electrode is the premise of inspecting. The feature points' theoretical coordinate information of the tool-electrode is extracted from the theoretical model of the tool-electrode. With these feature points as the target points, probe is drove by the axes to measure the actual coordinate information of them. Through the comparison between the theoretical coordinate information and the actual coordinate information, the accuracy of the tool-electrode can be inspected.

Because the tool-electrode's working surfaces are free-form, mesh generation method is adopted to choose the feature points. The grid nodes of the mesh are the feature points. When the curvature change of the mesh line is not high, the feature points are chosen in the way of equal interval. When the curvature change is high, the feature points are chosen in the way of variable interval.

The flow of extracting the information of the feature points is shown in figure 3 . In order to analyze the accuracy of the tool-electrode, the data information should not only include the coordinates, but also include the direction consines. The data format of the point is showed in formula (1). $X_{n}, y_{n}, z_{n}$ are the coordinates value, and $\cos \alpha_{n}, \cos \beta_{n}, \cos \gamma_{n}$ are the direction cosines. The function is realized through the secondary development of the UG. 


$$
P_{n}=\left[x_{n}, y_{n}, z_{n}, \cos \alpha_{n}, \cos \beta_{n}, \cos \gamma_{n}\right]
$$

\section{Planning the inspecting path}

The purpose of planning the inspecting path is to establish the moving track of the probe. Reasonable track can improve efficiency and accuracy of inspecting. Figure 4 is a kind of tool-electrode. It has 4 working surfaces. It has to plan the inspecting path of a surface firstly, and then plan the whole path from the whole scope of the tool-electrode.

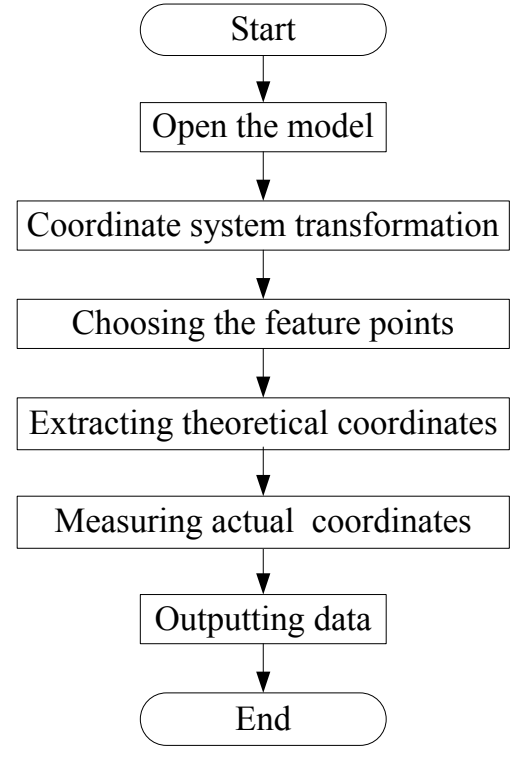

Fig.3. The flow of extracting the information of the feature points

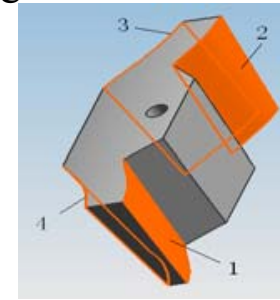

(a) Model of the tool-electrode

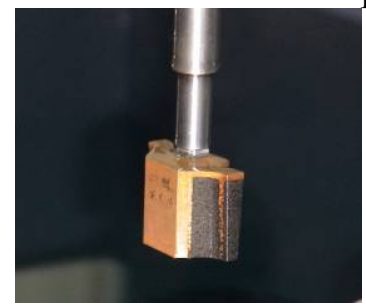

(b) Real object of the tool-electrode

Fig.4. Model of tool-electrode

Figure 5 is the inspecting path of the tool-electrode. Picture (a) is the path of a surface. Picture (b) is the whole path of the tool-electrode. The path of the surface is the combination of the path of the curve. $N_{i j}$ is serial number of the section. Letter " $i$ " expresses the surface inspected, and letter " $j$ " expresses the section number of the surface. $P_{i j k}$ is the feature point. Letter " $k$ " expresses the point number of the section. After the last point of a section has been inspected, the probe moves to the start point of the next section and inspect it. Because surface 1 and surface 2 are at the same direction, and surface 3 and surface 4 are at the same direction, when surface 1 and surface 2 have been inspected, the tool-electrode has to rotate to make surface 3 and surface 4 face to the electrode. In order to avoiding the impacting between the tool-electrode and the probe during the rotating procedure, after figure 2 has been inspected, the probe returns to the start point. So the whole inspecting path of the tool-electrode is: start point, surface 1, surface 2, start point, surface 3, surface 4 , and start point.

The data information that has been extracted is not the information of the feature point. It is the information of the probe center. So it has to be processed with radius compensation. Formula 2 is the transforming formula.

$$
\left\{\begin{array}{l}
x_{i j k}^{P}=x_{i j k} \pm r \cos \alpha_{i j k} \\
y_{i j k}^{P}=y_{i j k} \pm r \cos \beta_{i j k}
\end{array}\right.
$$



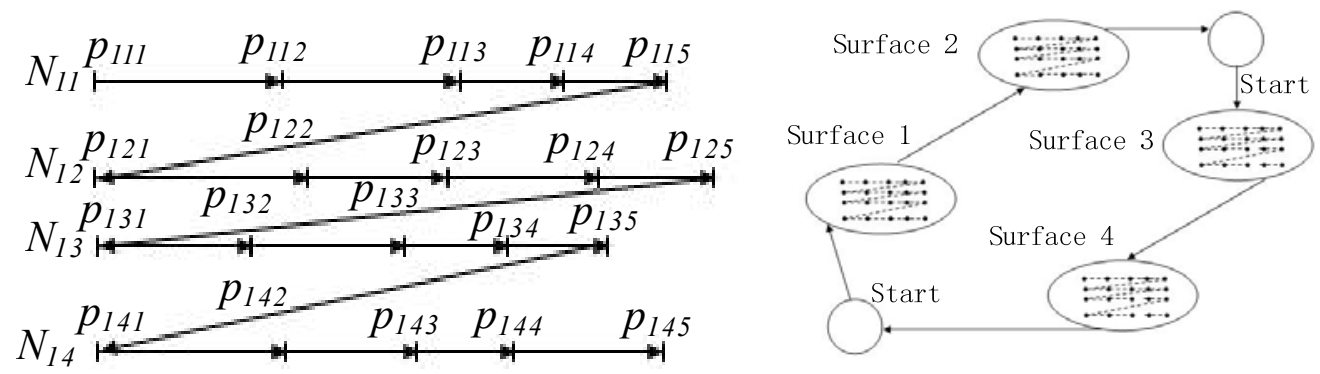

(a) Path of a surface

(b) Whole path of the tool-electrode

Fig.5. Inspecting path of the tool-electrode

\section{Accuracy evaluation of the tool-electrode}

The accuracy evaluation of the tool-electrode can through the error between the theoretical curve and the actual curve. It is the distance between the two curves at the actual feature point, as shown in picture (a) of figure 6. $P_{c}$ is actual feature point, $M_{a}$ is corresponding point. $\vec{n}_{p}$ is normal vector, $\vec{b}$ is its direction number. The coordinates of $P_{c}$ described in vector style are

$\vec{P}_{c}=\left\{x_{0}, y_{0}, z_{0}\right\}$

$\vec{b}=\left\{n_{x}, n_{y}, n_{z}\right\}$

The normal equation is:

$p(u)=\vec{P}_{c}+\vec{b} u$

$u$ is parameter.

$\overrightarrow{M_{a}}=\left\{m_{x}, \quad m_{y}, m_{z}\right\}$

$\overrightarrow{b^{\prime}}=\left\{m_{x}-x_{0}, \quad m_{y}-y_{0}, \quad m_{z}-z_{0}\right\}$

$p(u)=\vec{P}_{c}+\vec{b}^{\prime} u$

Because $M_{a} P_{c}$ and $\vec{n}_{p}$ are on the same line,

$\left(\vec{P}_{c} \times \vec{b}\right)\left(\vec{P}_{c}+\vec{b}^{\prime} u\right)=0 \quad u \in[0,1]$

Through the expansion and the simplification, formula 10 can be gotten as follow:

$\left\{\begin{array}{l}f(u)=f_{x}(u)+f_{y}(u)+f_{z}(u)=0 \\ f_{x}(u)=\left[\left(m_{z}-z_{0}\right) y_{0}-\left(m_{y}-y_{0}\right) z_{0}\right] \cdot p_{x}(u) \\ f_{y}(u)=\left[\left(m_{x}-x_{0}\right) z_{0}-\left(m_{z}-z_{0}\right) x_{0}\right] \cdot p_{y}(u) \\ f_{z}(u)=\left[\left(m_{y}-y_{0}\right) x_{0}-\left(m_{x}-x_{0}\right) y_{0}\right] \cdot p_{z}(u) \\ u \in[0,1]\end{array}\right.$

It is the univariate cubic equation of $u$, and it can be solved with one dimension optimization. Then, optimized point can be determined.

The direction of the error is shown in picture (b) of figure $6 . N$ is a point of the tool-electrode on the line of $\vec{n}_{p}$. If $M_{a}$ is outside the tool-electrode, $\overline{M_{a} N}>\overline{P_{c} N}$, error is positive. If $M_{a}$ is inside the tool-electrode, $\overline{M_{a} N}<\overline{P_{c} N}$, error is negative.

In picture (c) of figure $6, P_{i j k}$ is actual feature point, $P_{i j k}^{\prime}$ is optimized point solved form formula 10. $\overline{P_{i j k}^{\prime \prime} P_{i j k}}$ is the error between the theoretical curve and the actual curve. It can be 
obtained with following formulas.

$\overline{P_{i j k}^{\prime \prime} P_{i j k}} \doteq \overline{P_{i j k}^{*} P_{i j k}}$
$\overline{P_{i j k}^{*} P_{i j k}}=\overline{P_{i j k}^{\prime} P_{i j k}} \cdot \cos \alpha$
$\overline{P_{i j k}^{\prime} P_{i j k}}=\sqrt{\left(x_{i j k}-x_{i j k}^{\prime}\right)^{2}+\left(y_{i j k}-y_{i j k}^{\prime}\right)^{2}+\left(z_{i j k}-z_{i j k}^{\prime}\right)^{2}}$
$\cos \alpha=\frac{\left(x_{i j k}-x_{i j k}^{\prime}\right) \cdot n_{p x}+\left(y_{i j k}-y_{i j k}^{\prime}\right) \cdot n_{p y}+\left(z_{i j k}-z_{i j k}^{\prime}\right) \cdot n_{p z}}{\sqrt{\left(x_{i j k}-x_{i j k}^{\prime}\right)^{2}+\left(y_{i j k}-y_{i j k}^{\prime}\right)^{2}+\left(z_{i j k}-z_{i j k}^{\prime}\right)^{2}}}$

Because the points are in the same section,

$z_{i j k}=z_{i j k}^{\prime}$

Formula 13 can be simplified into formula 15:

$\left\{\begin{array}{l}\left|\overline{P_{i j k}^{\prime} P_{i j k}}\right|=\sqrt{\left(x_{i j k}-x_{i j k}^{\prime}\right)^{2}+\left(y_{i j k}-y_{i j k}^{\prime}\right)^{2}} \\ \cos \alpha=\frac{\left(x_{i j k}-x_{i j k}^{\prime}\right) \cdot n_{p x}+\left(y_{i j k}-y_{i j k}^{\prime}\right) \cdot n_{p y}}{\sqrt{\left(x_{i j k}-x_{i j k}^{\prime}\right)^{2}+\left(y_{i j k}-y_{i j k}^{\prime}\right)^{2}}}\end{array}\right.$

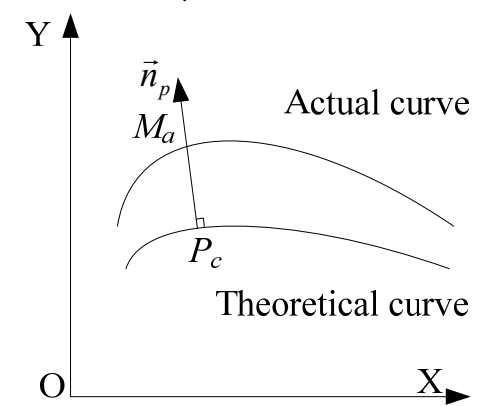

(a) Error between the theoretical curve

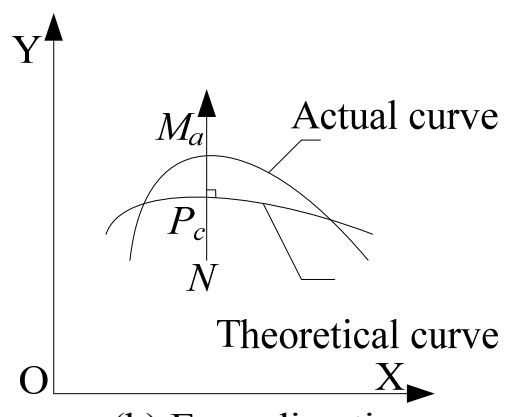

(b) Error direction

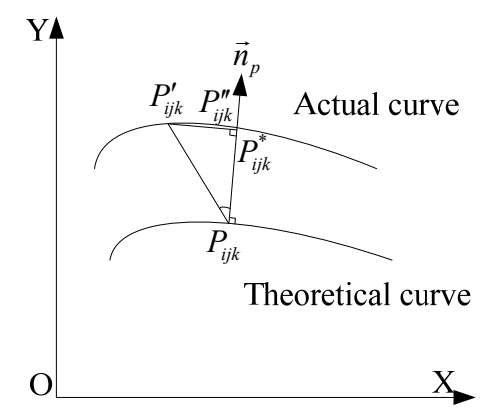

(c) Error at the feature point

Fig.6. Inspecting path of the tool-electrode

\section{Experiment results}

The following tables are a part of experimental data. Table 1 is the partial data of theoretical points, table 2 is partial data of optimized points, and table 3 is the deviation. The accuracy of the tool-electrode can be evaluated with these data. According to the permissible deviation, the eligibility of the tool-electrode can be determined.

Table 1 Partial data of theoretical points $(\mathrm{mm})$

\begin{tabular}{ccccccc}
\hline No. $(i)$ & $x_{i}$ & $y_{i}$ & $z_{i}$ & $\cos \alpha_{i}$ & $\cos \beta_{i}$ & $\cos \gamma_{i}$ \\
\hline 1 & 5.1873 & 12.162 & 37.0000 & 0.9321 & -0.2609 & 0.0000 \\
2 & 3.2906 & 10.1878 & 37.0000 & 0.9107 & -0.3135 & 0.0000 \\
3 & 1.818 & 8.0607 & 37.0000 & 0.9221 & -0.3857 & 0.0000 \\
4 & 0.3521 & 6.5521 & 37.0000 & 0.9235 & -0.4789 & 0.0000 \\
5 & 0.6651 & 6.3701 & 27.0000 & 0.9318 & -0.3002 & 0.0000 \\
6 & 1.8796 & 8.0976 & 27.0000 & 0.9105 & -0.3457 & 0.0000 \\
7 & 3.015 & 10.8216 & 27.0000 & 0.9157 & -0.3108 & 0.0000 \\
8 & 5.2077 & 12.3097 & 27.0000 & 0.9233 & -0.3778 & 0.0000 \\
\hline
\end{tabular}


Table 2 Partial data of optimized points (mm)

\begin{tabular}{cccc}
\hline No. $(i)$ & $x_{i}$ & $y_{i}$ & $z_{i}$ \\
\hline 1 & 5.182 & 12.1601 & 37.0000 \\
2 & 3.2915 & 10.1843 & 37.0000 \\
3 & 1.8177 & 8.0598 & 37.0000 \\
4 & 0.3552 & 6.5579 & 37.0000 \\
5 & 0.66 & 6.3673 & 27.0000 \\
6 & 1.8781 & 8.0925 & 27.0000 \\
7 & 3.01 & 10.8277 & 27.0000 \\
8 & 5.2067 & 12.3028 & 27.0000 \\
\hline Table 3 & Deviation of partial points $(\mathrm{mm})$ \\
\hline No. $(i)$ & $\Delta x_{i}$ & $\Delta y_{i}$ & $\Delta L_{i}$ \\
\hline 1 & -0.0053 & -0.0019 & 0.0056 \\
2 & 0.0009 & -0.0035 & 0.0036 \\
3 & -0.0003 & -0.0009 & 0.0009 \\
4 & 0.0031 & 0.0058 & 0.0065 \\
5 & -0.0051 & -0.0028 & 0.0058 \\
6 & -0.0015 & -0.0051 & 0.0053 \\
7 & -0.005 & 0.0061 & 0.0078 \\
8 & -0.001 & -0.0069 & 0.0069 \\
\hline
\end{tabular}

\section{Conclusion}

Through the experiment validation, the design and the realization of the adjusting and inspecting equipment are suitable for EDM. With this equipment, at the same time of machining, the adjusting and inspecting can be accomplished successively. This not only avoids the applying of unqualified tool-electrode, but also saves lots of unproductive time. So the work has important practical significance and application value. In the future work, in order to improve the inspecting accuracy, surface fitting can be used in error analysis.

\section{Acknowledgement}

In this paper, the research was sponsored by the National High-tech. R\&D Program (Project No. 2009AA044201).

\section{References}

[1] Ming Zhou, Fuzhu Han, Yongxain Wang, Isago Soichiro. Assessment of the dynamical properties in EDM process - detecting deterministic nonlinearity of EDM process [J]. The International Journal of Advanced Manufacturing Technology, 200944 (1) 91-99.

[2] Gunawan Setia Prihandana, Muslim Mahardika, M. Hamdi, Kimiyuki Mitsui. Effect of low-frequency vibration on workpiece in EDM processes [J]. Journal of Mechanical Science and Technology, 201125 (5) 1231-1234.

[3] Shinya Hayakawa, Masahiro Takahashi, Fumihiro Itoigawa, et al.. Study on EDM phenomena with in-process measurement of gap distance [J]. Journal of Materials Processing Technology, 2004 149 (6): 250-255.

[4] Spiros Zinelis, Youssef S. Al Jabbari, Andrew Thomas, Nick Silikas, George Eliades. Multitechnique characterization of CPTi surfaces after electro discharge machining (EDM) [J]. Clinical Oral Investigations, 201418 (1) 67-75.

[5] L. Li, X. T. Wei, Z. Y. Li, X. Cheng. Corrosion Resistance Analysis of Sintered NdFeB 
Magnets Using Ultrasonic-Aided EDM Method [J]. Journal of Materials Engineering and Performance, 201524 (1) 536-542. 